\title{
Progesterona para el tratamiento del trauma craneoencefálico grave: una revisión sistemática y metaanálisis
}

\author{
William Flórez Perdomo*, Miguel Durán†, Edgar F Laiseca†, Mario F Quesada ${ }^{\ddagger}$
}

\section{Resumen}

Objetivo: revisar sistemáticamente la evidencia sobre la administración de progesterona tras un trauma craneoencefálico grave en adultos y su relación con mortalidad y pronóstico neurológico.

Criterios de inclusión: ensayos clínicos aleatorizados que incluyan a pacientes adultos mayores de 18 años, haber sufrido un traumatismo craneal grave (Glasgow $\leq 8$ ), donde se compare la administración de progesterona vs grupo control (placebo o no administración).

Método: se realizó la búsqueda en las siguientes bases de datos: MEDLINE, the Central Register of Controlled Trials (CENTRAL); PubMed, HINARI, EMBASE; Cochrane Injuries group y lista de referencia de los artículos.

Resultados: no hubo reducción de la mortalidad comparado con el grupo control (RR 0,93, IC95\% 0,79-1,10 p= 0,41), no hubo diferencias entre progesterona y el grupo control en desenlaces neurológicos positivos ni negativos (RR 1,07, IC95\% 0,97-1,17 $p=0,20 ; \operatorname{RR} 0,94$, IC 95\% 0,81-1,08 $p=0,27$ ), respectivamente.

Conclusiones: no se encontró evidencia respecto a que la administración de progesterona posterior a un traumatismo craneoencefálico reduzca la mortalidad o mejore desenlaces neurológicos, aunque se necesitan más estudios de buena calidad para extraer conclusiones definitivas.

Palabras clave: Lesiones traumáticas del encéfalo

Progesterona

Metaanálisis

Key words: $\quad$ Traumatic brain injuries

Progesterone

Meta-Analysis

\footnotetext{
* Departamento de Urgencias, IPS Clínica Sahagún, Sahagún - Córdoba, Colombia.

† Médico general. Universidad Surcolombiana.

‡ Fundación Universitaria Navarra.

Correspondencia: William A Flórez Perdomo. Carrera 14 \# 10-41, Sahagún-Córdoba, Colombia. Correo electrónico: William-florez@hotmail.com Los autores declaran no tener conflicto de intereses.

Recibido: 22/7/19

Aprobado: 23/10/19
} 


\section{Introducción}

La lesión cerebral traumática es una de las principales causas de mortalidad y discapacidad ${ }^{(1))}$, se estima que 57 millones de personas han sido hospitalizadas alrededor del mundo con algún grado de traumatismo craneoencefálico y al año representan aproximadamente 10 millones de hospitalizaciones y muertes ${ }^{(2,3)}$. Esta es una condición que tiene un alto impacto socioeconómico trayendo consigo altos costos en salud; se estima que en Estados Unidos se ha incrementado hasta 76,1 millones de dólares ${ }^{(3-5)}$.

En la fisiopatología del trauma craneoencefálico se producen dos tipos de daños neurológicos, al momento del trauma (primaria), causado por mecanismos como edema cerebral, formación de radicales libres o liberación de inflamatorios (lesión secundaria) ${ }^{(6,7)}$, por lo que se han estudiado diversas medidas farmacológicas para prevenir o reducir los efectos de los mecanismos secundarios, como corticoides y sulfato de magnesio, los cuales no han demostrado beneficios ${ }^{(8,9)}$. Es importante buscar un medicamento seguro y clínicamente efectivo que funcione como neuroprotector tras recibir un traumatismo craneoencefálico, y la progesterona parece ser buena candidata.

La progesterona es una hormona sexual con funciones neuroactivas y esteroideas que tiene acciones sobre el sistema nervioso central ${ }^{(10)}$. Esta hormona está presente en el cerebro de hombres y mujeres en concentraciones pequeñas, aproximadamente iguales ${ }^{(11)}$.

Ciertamente, hay una amplia distribución de receptores de progesterona con amplia acción de la hormona gonadal y la diversidad de funciones, como el crecimiento y diferenciación neuronal ${ }^{(12)}$, su supervivencia ${ }^{(13)}$, plastici$\operatorname{dad}^{(14)}$, y modulan funciones cognitivas ${ }^{(15)}$.

En modelos experimentales in vitro y estudios con animales sugieren que el tratamiento posterior a la lesión con progesterona disminuye el edema cerebral, atenúa el daño de los radicales libres y reduce la pérdida neuronal en modelos de animales con trauma craneoencefáli$\operatorname{co}^{(16)}$.

Nosotros conducimos una revisión sistemática para que nos muestre la evidencia científica existente sobre la administración de progesterona en pacientes adultos con traumatismo craneoencefálico grave y su impacto con la mortalidad y pronóstico neurológico.

\section{Método}

El esquema a seguir será de acuerdo a las recomendaciones de metaanálisis y revisiones sistemáticas de la declaración PRISMA ${ }^{(17)}$, para la presentación de revisiones sistemáticas y metaanálisis, y el manual Cochrane de revisiones sistemáticas y metaanálisis ${ }^{(18)}$.

\section{Búsqueda}

Se realizó búsqueda de ensayos clínicos aleatorizados (RCT's) a través de PUBMED (hasta 2019); Registro Especializado Cochrane Injuries Group (hasta 2019); Registro Cochrane Central de Ensayos Controlados (The Cochrane Library) (hasta 2019); MEDLINE (Ovid) hasta 2019; EMBASE (Ovid); PubMed (http://www.ncbi. nlm.nih.gov/sites/entrez) (hasta 2019); además de la lista de referencia de los estudios incluidos y otros datos relevantes de los estudios potencialmente elegibles. Nosotros realizamos la búsqueda en internet a través de Google Scholar (www.googlescholar.com) para búsqueda de literatura gris y de estudios en revistas no indexadas y la base de datos de Science Direct (www.sciencedirect.com) con los términos seleccionados en la estrategia de búsqueda.

La búsqueda se construyó usando términos y descriptores del Medical Subject Heading (MeSH), todos combinados con operadores booleanos.

Brain Injuries (*drug therapy; *mortality); Disability Evaluation; Glasgow Coma Scale; Injury Severity Score; Intracranial Pressure (drug effects); Neuroprotective Agents (adverse effects; *therapeutic use); Progesterone (adverse effects; *therapeutic use); Randomized Controlled Trials, Humans.

La estrategia de búsqueda completa se encuentra en el anexo A.

\section{Criterios de inclusión}

Se incluyeron ensayos clínicos aleatorizados que evalúan pacientes con trauma craneoencefálico grave (escala de Glasgow $\leq 8$ ), que incluyan población adulta ( $>18$ años de edad), que compare el uso de progesterona versus placebo, sin tener en cuenta dosis, vía y velocidad de administración, cuyos resultados clínicos sean mortalidad y pronóstico neurológico.

\section{Análisis estadístico}

De manera individual y separada se extrajeron los siguientes datos: mortalidad, pronóstico (buen pronóstico y mal pronóstico). Se contactaron a los autores por datos faltantes. Las dudas se aclararon mediante consenso de los autores. El análisis estadístico se realizó a través del riesgo relativo (RR) con un intervalo de confianza de 95\% (IC95\%) con la metodología Mantel-Haenszel para variables dicotómicas, con un modelo de análisis de efecto fijado calculado mediante el software Review Manager 5.3. La heterogeneidad se evaluó mediante el cálculo de chi cuadrado (I2), siendo por encima de 50\% una alta heterogeneidad de los estudios incluidos en el análisis. La variable primaria a evaluar fue la mortalidad, las secundarias buen y mal pronóstico neurológico. 


\section{Evaluación de riesgo de sesgo}

Las revisiones sistemáticas fueron evaluadas por dos autores de manera independiente, posteriormente se realiza consenso entre los revisores para la evaluación de riesgo de sesgo de los estudios incluidos, de acuerdo a los lineamientos del manual Cochrane para revisiones sistemáticas (Centro Cochrane Iberoamericano, traductores. Manual Cochrane de Revisiones Sistemáticas de Intervenciones, versión 5.3, actualizada en octubre de 2016, internet). Barcelona: Centro Cochrane Iberoamericano; 2012. (http://www.cochrane.es/?q=es/node/269), cuyo modelo se muestra a detalle en anexo B.

Los siguientes dominios fueron evaluados y ubicados en las siguientes categorías dependiendo de su validez y del riesgo de sesgo en: bajo riesgo de sesgo, riesgo de sesgo no claro o alto riesgo de sesgo:

- Generación de secuencia.

- Ocultamiento de la asignación.

- Cegamiento de los participantes y de los evaluadores.

- Datos incompletos de los resultados.

- Notificación selectiva de los resultados y otros tipos de sesgo.

Planeamos evaluar el sesgo de publicación por gráficos de embudo y lineal pruebas de regresión (Eger Test), sin embargo, hubo muy pocos estudios incluidos para permitir un análisis significativo. Evaluaremos los sesgos de publicación en futuras versiones de la revisión, si hay diez o más estudios incluidos en el metaanálisis.

\section{Resultados}

Tras realizar la búsqueda sistemática de la información, siguiendo nuestra estrategia, se identificaron 318 citas bibliográficas, de las cuales 18 se consideraron potencialmente elegibles sobre la base de título o el resumen, o ambos, y se obtuvieron los textos completos. Después de una revisión del texto completo, 13 ensayos se consideraron elegibles, cinco fueron descartados por no cumplir criterios de inclusión ${ }^{(19-23)}$ y siete reunieron los criterios de inclusión para la revisión (figura 1; anexo $C)^{(24-30)}$.

Se evaluó el riesgo de sesgo para los distintos estudios escogidos mediante la metodología descrita y se encontró que el $20 \%$ de los estudios presenta alto riesgo y $10 \%$ de riesgo no claro de sesgo de reporte selectivo, sesgo de asignación y cegamiento de los resultados, $20 \%$ riesgos sobre el cegamiento de los participantes, $30 \%$ de riesgo de otros tipos de sesgo. Ningún estudio tuvo algún riesgo de sesgo. Dos de los estudios elegidos

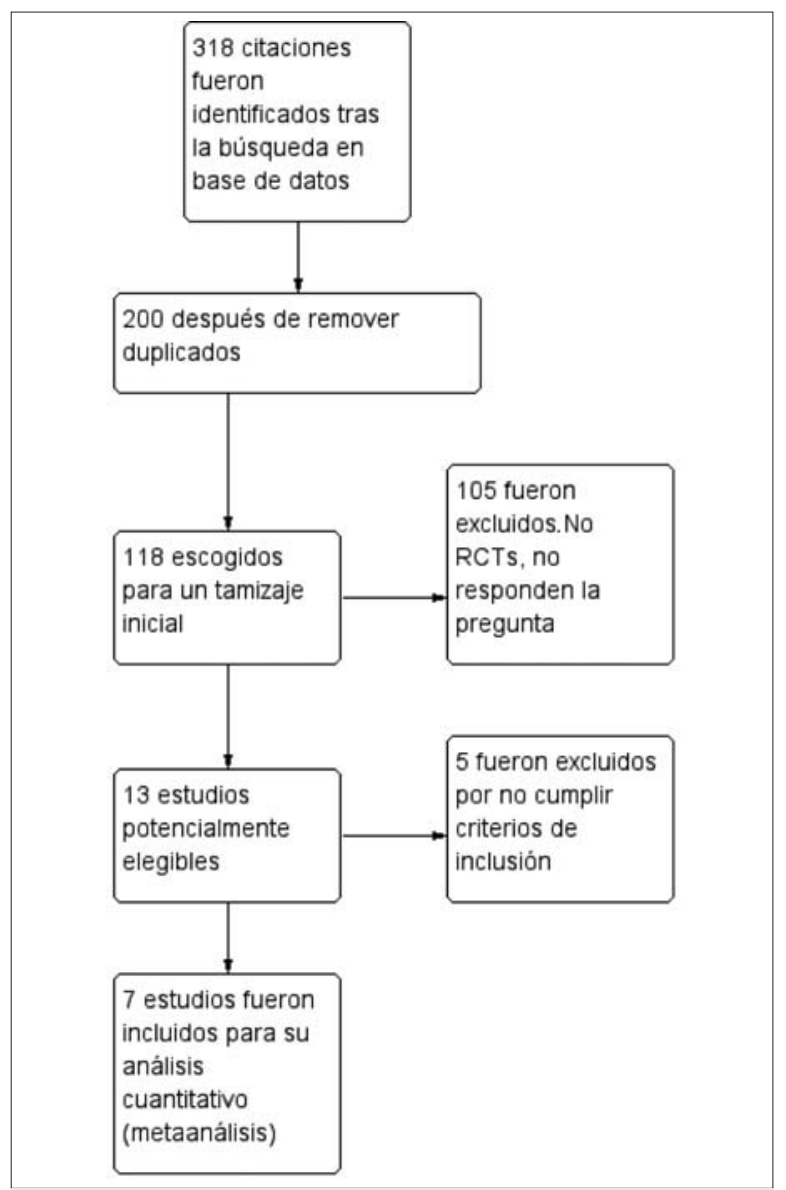

Figura 1. Diagrama de flujo (PRISMA).

están libres de riesgo de sesgo (SYNAPSE y NETT) (figuras 2 y 3$)^{(25,29)}$

Se escogieron siete estudios para el análisis final que involucran a 1.792 pacientes. En todos los estudios se evaluaron las variables primarias y secundarias a seis meses mediante la escala de Glasgow evolutiva.

No hubo reducción de la mortalidad en pacientes en quienes se administró progesterona posterior al trauma comparado con el grupo control (RR 0,93, IC95\% $0,79-1,10 \mathrm{p}=0,41)$ sin significancia estadística, hubo evidencia de alta heterogeneidad entre los estudios $\left(\mathrm{X}^{2}=\right.$ $\left.13,4 \mathrm{df}=6 \mathrm{I}^{2}=55 \% \mathrm{p}=0,04\right)$ (tabla 1 y figura 4 ).

El protocolo de cuidado neurocrítico con administración de progesterona no muestra diferencia significativa en comparación a control en el contexto de desenlaces neurológicos favorables (discapacidad moderada a buena recuperación evaluada por escala de Glasgow evolutiva 5 - 6) (RR 1,07, IC95\% 0,97-1,17 p= $0,20)\left(X^{2}=17,46 \mathrm{df}=6 \mathrm{I}^{2}=66 \% \mathrm{p}=0,08\right)($ figura 5), se evidencia alta heterogeneidad de los estudios que pueden afectar el análisis estadístico de este desenlace clínico y no favorables (estado vegetativo a discapacidad 


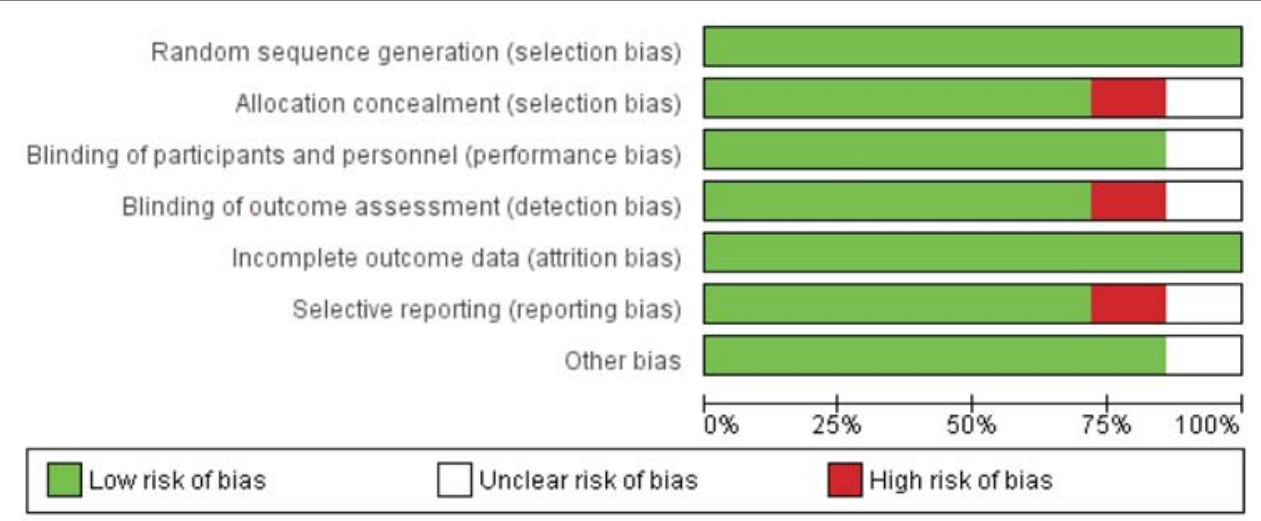

Figura 2. Gráfico de riesgo de sesgo: los juicios de los autores de la revisión sobre cada elemento de riesgo de sesgo se presentan como porcentajes en todos los estudios incluidos.

severa, GOS-E 1-3) (RR 0,94, IC 95\% 0,81-1,08 p= $0,27)\left(X^{2}=7,64 \mathrm{df}=6 \mathrm{I}^{2}=21 \% \mathrm{p}=0,27\right)$ (figura 6).

\section{Discusión}

La administración de progesterona posterior al trauma craneoencefálico muestra muchos avances a nivel experimental $^{(31)}$. Por ser liposuble atraviesa fácilmente la barrera hematoencefálica y alcanza el equilibrio con el plasma dentro de una hora de la administración ${ }^{(32)}$.

A pesar de los extensos datos preclínicos y dos prometedores ensayos de centro único ${ }^{(25,26,33)}$, progesterona no fue asociada con cualquier beneficio sobre el placebo, según lo medido por el puntaje GOS-E a los seis meses en este gran ensayo clínico multicéntrico. Los grupos fueron bien equilibrados para la gravedad de la lesión, y tanto el análisis por intención de tratar y a priori planeado. El análisis de la población objetivo fue congruente en mostrar ningún efecto de tratamiento. Se han realizado muchas pruebas negativas o resultados no concluyentes en las arduas búsquedas de un tratamiento para traumatismo craneoencefálico. De los diversos ensayos clínicos, ninguno ha tenido éxito ${ }^{(34)}$.

En nuestra revisión sistemática y metaanálisis no encontramos evidencia de que la progesterona pudiera disminuir la mortalidad a mortalidad general (RR 0,93, IC95\% 0,79-1,10 p= 0,41), ni el pronóstico neurológico no favorable (estado vegetativo a discapacidad severa, GOS-E 1-3) (RR 0,94, IC95\% 0,81-1,08 p= 0,27).

En el año 2016 fue publicada en la Cochrane library una revisión sistemática (Ma J y colaboradores) ${ }^{(10)}$, donde fueron incluidos cinco estudios donde se comparó el uso de progesterona con el mantenimiento de normotermia en niños y adultos (sin límite de edad) que requirieron hospitalización posterior a un trauma craneoencefálico. En esta revisión no se discriminó la severidad del trauma y se incluyeron estudios donde se evaluaba esta

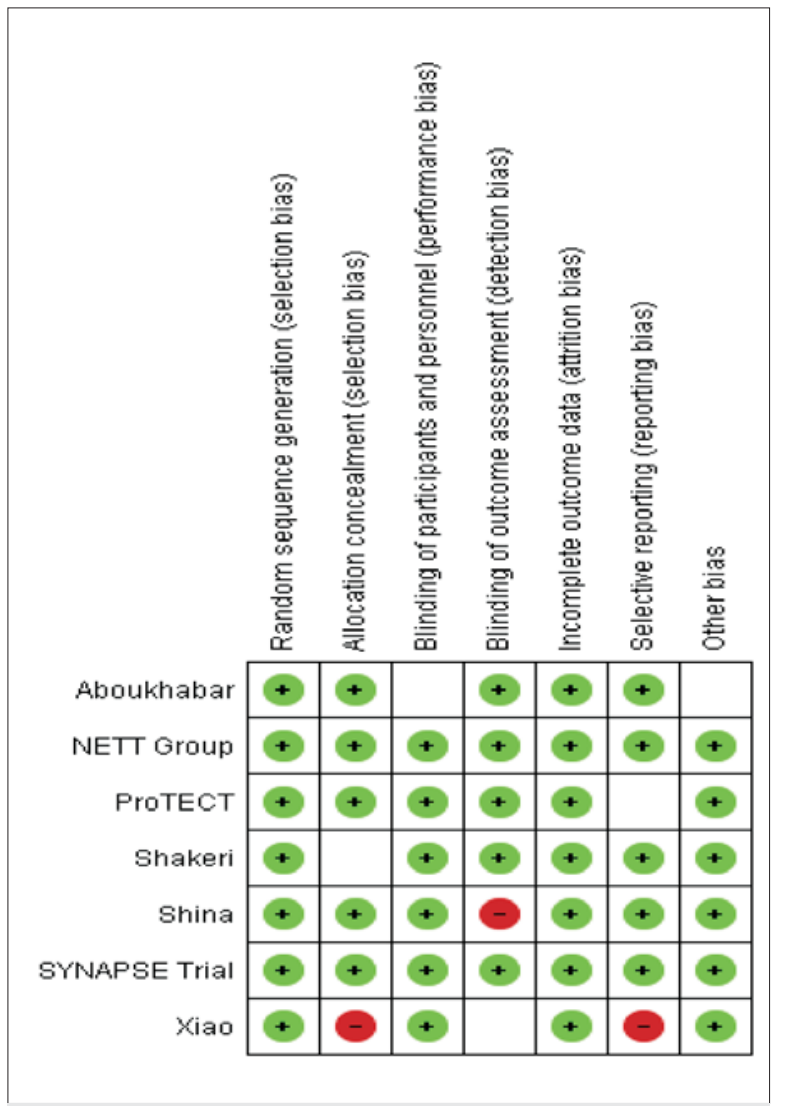

Figura 3. Resumen del riesgo de sesgo: revisión de las opiniones de los autores sobre cada elemento de riesgo de sesgo para cada estudio incluido.

técnica en pacientes con TCE moderado o grave, donde no se encontraron diferencias significativas con el grupo control, en esta revisión, a diferencia de las que conducimos, se excluyeron fármacos como el acetato de medroxiprogesterona. 


\begin{tabular}{|c|c|c|c|c|c|c|c|c|c|}
\hline \multirow[b]{2}{*}{ Study or Subgroup } & \multicolumn{2}{|c|}{ Progesterona } & \multicolumn{2}{|c|}{ Control } & \multirow[b]{2}{*}{ Weight } & \multirow{2}{*}{$\begin{array}{c}\text { Risk Ratio } \\
\text { M-H, Fixed, } 95 \% \mathrm{Cl}\end{array}$} & \multirow{2}{*}{\multicolumn{3}{|c|}{$\begin{array}{c}\text { Risk Ratio } \\
\text { M-H, Fixed, } 95 \% \mathrm{Cl}\end{array}$}} \\
\hline & Events & Total & Events & Total & & & & & \\
\hline Aboukhabar & 8 & 50 & 7 & 50 & $3.3 \%$ & $1.14[0.45,2.91]$ & & & \\
\hline NETT Group & 29 & 74 & 19 & 74 & $9.0 \%$ & $1.53[0.94,2.47]$ & & - & \\
\hline ProTECT & 10 & 77 & 7 & 23 & $5.1 \%$ & $0.43[0.18,0.99]$ & & & \\
\hline Shakeri & 4 & 29 & 10 & 31 & $4.6 \%$ & $0.43[0.15,1.21]$ & & & \\
\hline Shina & 5 & 23 & 7 & 23 & $3.3 \%$ & $0.71[0.27,1.93]$ & & & \\
\hline SYNAPSE Trial & 131 & 591 & 131 & 588 & $62.4 \%$ & $0.99[0.80,1.23]$ & & & \\
\hline Xiao & 15 & 82 & 25 & 77 & $12.2 \%$ & $0.56[0.32,0.99]$ & 1 & & \\
\hline Total $(95 \% \mathrm{Cl})$ & & 926 & & 866 & $100.0 \%$ & $0.93[0.79,1.10]$ & 4 & & \\
\hline Total events & 202 & & 206 & & & & & & \\
\hline $\begin{array}{l}\text { Heterogeneity: } \mathrm{Chi}^{2}= \\
\text { Test for overall effect }\end{array}$ & $\begin{array}{l}13.40, d f= \\
Z=0.83\end{array}$ & $\begin{array}{l}6(P=0 \\
=0.41)\end{array}$ & $0.04) ; 1^{2}=$ & $55 \%$ & & & $\begin{array}{ccc}0.01 & 0.1 \\
& \text { Favorable Progesterona }\end{array}$ & $\begin{array}{c}10 \\
\text { Favorable Control }\end{array}$ & 100 \\
\hline
\end{tabular}

Figura 4. Forest plot de comparación: 1 progesterona vs control, desenlace: 1.1 mortalidad.

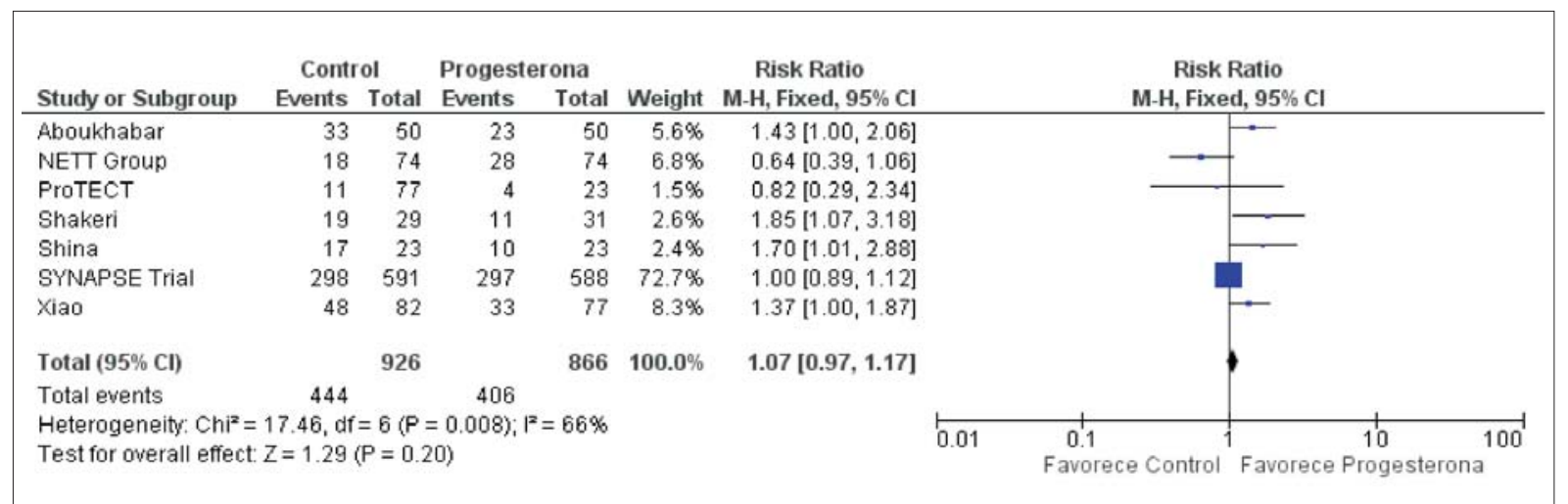

Figura 5. Forest plot de comparación: 1 progesterona vs control, desenlace: 1.2 buen pronóstico neurológico (Glasgow Outcome: moderada discapacidad a buena recuperación).

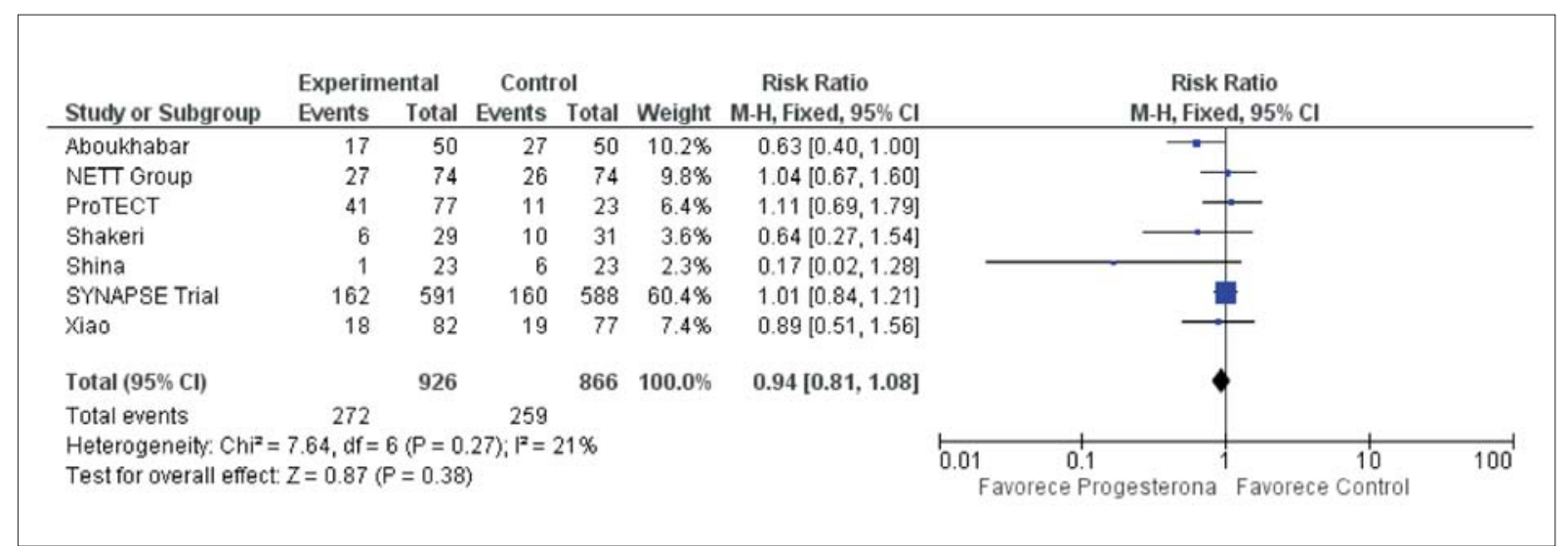

Figura 6. Forest plot de comparación: 1 progesterona vs control, desenlace: 1.3 mal pronóstico neurológico (Glasgow Outcome: discapacidad severa a estado vegetativo/muerte).

Este año se publicó un metaanálisis publicado en Drug Design, Development and Therapy donde se evaluaron ocho ensayos clínicos con un total de 2.251, con una disminución significativa en mortalidad y pronóstico neurológico adverso a tres meses del seguimiento; sin embargo, no hubo diferencia significativa a los seis meses posteriores al traumatismo, se estratificaron los resultados sobre la vía de administración intramuscular e intravenosa, y los efectos benéficos solo se mostraron en el grupo donde se administró progesterona por vía intramuscular ${ }^{(35)}$. 
Tabla 1. Resumen de principales variables (se incluyen primarias y secundarias).

\begin{tabular}{lcccc}
\hline Variables & Estudios & Participantes & Método estadístico & Estimación de efecto \\
\hline 1.1 Mortalidad & 7 & 1.792 & $\begin{array}{l}\text { Riesgo relativo (M-H, } \\
\text { efectos fijos IC95\%) }\end{array}$ & $0,93(0,79,1,10)$ \\
$\begin{array}{l}1.2 \text { Buen pronóstico neurológico } \\
\begin{array}{l}\text { (Glasgow Outcome: moderada } \\
\text { discapacidad a buena recuperación) }\end{array}\end{array}$ & 7 & 1.792 & $\begin{array}{l}\text { Riesgo relativo (M-H, } \\
\text { efectos fijos IC95\%) }\end{array}$ & $1,13(0,94,1,37)$ \\
$\begin{array}{l}1.3 \text { Mal pronóstico neurológico } \\
\text { (Glasgow Outcome: discapacidad } \\
\text { severa a estado vegetativo/muerte) }\end{array}$ & 7 & 1.792 & $\begin{array}{l}\text { Riesgo relativo (M-H, } \\
\text { efectos fijos IC95\%) }\end{array}$ & $0,94(0,81,1,08)$ \\
\hline
\end{tabular}

\section{Limitaciones}

Los trabajos muestran gran variación entre los protocolos utilizados en relación con la dosis y la vía de administración de progesterona, así como en la velocidad de infusión y el tiempo de aplicación de ésta después del trauma. Otros puntos débiles fueron la existencia de pocos estudios clínicos aleatorizados de calidad y la gran heterogeneidad de pacientes que se incluyen en los estudios.

Aunque algunos de los ensayos incluidos en esta revisión fueron bien realizados en términos de tamaño de muestra y minimización del sesgo, su utilidad para determinar los efectos del tratamiento con progesterona para el TBI agudo estaba limitada por la heterogeneidad de las poblaciones, la inconsistencia de las intervenciones y las limitaciones de los resultados medidos.

\section{Conclusiones}

No se encontró evidencia de que la administración de progesterona posterior a un traumatismo craneoencefálico pueda reducir la mortalidad o mejorar los desenlaces neurológicos; sin embargo, los hallazgos son limitados y la calidad de la evidencia es baja o moderada, por lo que se necesitan más estudios de calidad y de adecuado muestreo.

\section{Abstract}

Objective: to systematically review evidence on the administration of progesterone after a traumatic brain injury in adults and its relationship with mortality and neurological head prognosis.

Inclusion criteria: randomized clinical trials that include: patients older than 12 years old, having had an injury (Glasgow $<8$ ), comparing the administration of Progesterone versus the control group (placebo or no administration).
Methods: we searched the following databases: MEDLINE, the Central Register of Controlled Trials (CENTRAL); PubMed, HINARI, EMBASE; Cochrane Injury Group and reference list of articles.

Results: there was no reduction in mortality in patients in the control group (RR 0.93, 95\% CI 0.79-1.10 p $=0.41$ ), there were no differences between progesterone and the control group in favorable or adverse neurological outcomes (RR 1.07, 95\% CI: 0.97-1.17 p = 0.20, RR $0.94,95 \%$ CI: $0.81-1,08 p=0.27$ ), respectively.

Conclusions: there is no evidence that the administration of progesterone after a traumatic brain injury reduces or improves neurological results, although further good quality studies are required to obtain conclusive results.

\section{Resumo}

Objetivo: realizar uma revisão sistemática da evidência sobre a administração de progesterona depois de traumatismo crânio-encefálico grave em adultos e sua relação com a mortalidade e o prognóstico neurológico.

Critérios de inclusão: ensaios clínicos aleatorizados que incluam: pacientes adultos maiores de 18 anos, haver sofrido um traumatismo craniano grave (Glasgow $<8$ ) donde se compare a administração de progesterona versus grupo controle (placebo ou não administração).

Métodos: foi feita uma pesquisa bibliográfica nas seguintes bases de dados: MEDLINE, Central Register of Controlled Trials (CENTRAL), PubMed, HINARI, EMBASE, Cochrane Injuries Group e nas referências bibliográficas dos artigos.

Resultados: não foi observada uma redução da mortalidade comparada com o grupo controle (RR 0,93, IC del 95\%: 0,79-1,10 p=0,41), não foram observadas diferenças entre o grupo que recebeu progesterona e o grupo controle nos resultados neurológicos positivos ou negativos (RR 1,07, IC del 95\%: 0,97-1,17 p=0,20; RR 0,94, IC del 95\%: 0,81-1,08 p=0,27), respectivamente. 
Conclusões: não se encontrou evidência de que a administração de progesterona depois de um traumatismo crânio-encefálico reduza a mortalidade ou melhore os resultados neurológicos embora novos estudos de boa qualidade sejam necessários para chegar a conclusões definitivas.

\section{Bibliografía}

1. Ghajar J. Traumatic brain injury. Lancet 2000; 356(9233): 923-9.

2. Alarcon J, Rubiano A, Chirinos M, Valderrama A, Gich I, Bonfill X, et al. Clinical practice guidelines for the care of patients with severe traumatic brain injury: a systematic evaluation of their quality. J Trauma Acute Care Surg 2013; 75(2):311-9.

3. Langlois J, Rutland-Brown W, Thomas K. Traumatic brain injury in the United States: emergency department visits, hospitalizations, and deaths. Atlanta (GA): Centers for Disease Control and Prevention. National Center for Injury Prevention and Control, 2006.

4. Alali A, Fowler R, Mainprize T, Scales D, Kiss A, de Mestral C, et al. Intracranial pressure monitoring in severe traumatic brain injury: results from the American College of Surgeons Trauma Quality Improvement Program. J Neurotrauma 2013; 30(20):1737-46.

5. McGarry L, Thompson D, Millham F, Cowell L, Snyder P, Lenderking W, et al. Outcomes and costs of acute treatment of traumatic brain injury. J Trauma 2002; 53(6):1152-9.

6. Godoy D, Panhke P, Piñero G, Videtta W, Aguilera S. Cuidados intensivos en trauma craneoencefálico grave. Neuroc Colomb 2016; 23(3):193-234.

7. Guzman F. Pathophysiology of traumatic brain injury. Colomb Méd 2008; 39(Supl 3): 78-84

8. Alderson P, Roberts I. Corticosteroids for acute traumatic brain injury. Cochrane Database Syst Rev 2005; (1):CD000196.

9. Arango M, Bainbridge D. Magnesium for acute traumatic brain injury. Cochrane Database Syst Rev 2008; (4):CD005400.

10. Ma J, Huang S, Qin S, You C, Zeng Y. Progesterone for acute traumatic brain injury. Cochrane Database Syst Rev 2016; 12(12):CD008409.

11. Schumacher M, Baulieu E. Neurosteroids: synthesis and functions in the central and peripheral nervous systems. Ciba Found Symp 1995; 191:90-106.

12. Singh M, Su C. Progesterone-induced neuroprotection: factors that may predict therapeutic efficacy. Brain Res 2013; 1514:98-106

13. Toran-Allerand C. Sex steroids and the development of the newborn mouse hypothalamus and preoptic area in vitro. II. Morphological correlates and hormonal specificity. Brain Res 1980; 189(2):413-27.

14. Simpkins J, Green P, Gridley KE Singh M, de Fiebre N, Rajakumar G. Role of estrogen replacement therapy in me- mory enhancement and the prevention of neuronal loss associated with Alzheimer's disease. Am J Med 1997; 103(3A):19S-25S.

15. Singh M, Meyer E, Simpkins J. The effect of ovariectomy and estradiol replacement on brain-derived neurotrophic factor messenger ribonucleic acid expression in cortical and hippocampal brain regions of female Sprague-Dawley rats. Endocrinology 1995; 136(5):2320-4.

16. Asbury E, Fritts M, Horton J, Isaac W. Progesterone facilitates the acquisition of avoidance learning and protects against subcortical neuronal death following prefrontal cortex ablation in the rat. Behav Brain Res 1998; 97(1-2):99-106

17. Moher D, Liberati A, Tetzlaff J, Altman D. Preferred reporting items for systematic reviews and meta-analyses: the PRISMA statement. Version 2. BMJ 2009; 339:b2535.

18. Higgins J, Green S, eds. Cochrane Handbook for Systematic Reviews of Interventions: version 5.1.0, updated March 2011. London: The Cochrane Collaboration, 2011.

19. Mofid B, Soltani Z, Khaksari M, Shahrokhi N, Nakhaee $\mathbf{N}$, Karamouzian S, et al. What are the progesterone-induced changes of the outcome and the serum markers of injury, oxidant activity and inflammation in diffuse axonal injury patients? Int Immunopharmacol 2016; 32:103-110.

20. Xiao G, Wei J, Wu Z, Wang W, Jiang Q, Cheng J, et al. Clinical study on the therapeutic effects and mechanism of progesterone in the treatment for acute severe head injury. Zhonghua Wai Ke Za Zhi 2007; 45(2):106-8.

21. Aminmansour B, Nikbakht H, Ghorbani A, Rezvani M, Rahmani P, Torkashvand M, et al. Comparison of the administration of progesterone versus progesterone and vitamin $\mathrm{D}$ in improvement of outcomes in patients with traumatic brain injury: a randomized clinical trial with placebo group. Adv Biomed Res 2012; 1:58.

22. Raheja A, Sinha S, Samson N, Bhoi S, Subramanian A, Sharma $\mathbf{P}$, et al. Serum biomarkers as predictors of long-term outcome in severe traumatic brain injury: analysis from a randomized placebo-controlled Phase II clinical trial. J Neurosurg 2016; 125(3):631-41.

23. Wright D, Ritchie J, Mullins R, Kellermann A, Denson D. Steady-state serum concentrations of progesterone following continuous intravenous infusion in patients with acute moderate to severe traumatic brain injury. J Clin Pharmacol 2005; 45(6):640-8

24. Aboukhabar H, Abouelela A, Shaban S. Impact of progesterone administration on outcome in patients with severe traumatic brain injury. Res Opin Anesth Intensive Care 2017; 4(2):84-9.

25. Wright D, Yeatts S, Silbergleit R, Palesch Y, Hertzberg V, Frankel M, et al. Very early administration of progesterone for acute traumatic brain injury. N Engl J Med 2014; 371(26):2457-66.

26. Wright D, Kellermann A, Hertzberg V, Clark P, Frankel M, Goldstein F, et al. ProTECT: a randomized clinical trial of progesterone for acute traumatic brain injury. Ann Emerg Med 2007; 49(4):391-402, 402.e1-2. 
27. Shakeri M, Boustani M, Pak N, Panahi F, Salehpour F, Lotfinia I, et al. Effect of progesterone administration on prognosis of patients with diffuse axonal injury due to severe head trauma. Clin Neurol Neurosurg 2013; 115(10):2019-22.

28. Sinha S, Raheja A, Samson N, Goyal K, Bhoi S, Selvi A, et al. A randomized placebo-controlled trial of progesterone with or without hypothermia in patients with acute severe traumatic brain injury. Neurol India 2017; 65(6):1304-11

29. Skolnick B, Maas AI, Narayan R, Gerritsen R, MacAllister T, Wars J, et al. A clinical trial of progesterone for severe traumatic brain injury. N Engl J Med 2014; 371(26):2467-76.

30. Xiao G, Wei J, Yan W, Wang W, Lu Z. Improved outcomes from the administration of progesterone for patients with acute severe traumatic brain injury: a randomized controlled trial. Crit Care 2008; 12(2):R61.
31. Gibson C, Gray L, Bath P, Murphy S. Progesterone for the treatment of experimental brain injury; a systematic review. Brain 2008; 131(Pt 2):318-28.

32. Lancel M, Faulhaber J, Holsboer F, Rupprecht R. Progesterone induces changes in sleep comparable to those of agonistic GABAA receptor modulators. Am J Physiol 1996; 271(4 Pt 1):E763-72.

33. Deutsch E, Espinoza T, Atif F, Woodall E, Kaylor J, Wright D. Progesterone's role in neuroprotection, a review of the evidence. Brain Res 2013; 1530:82-105.

34. Loane D, Faden A. Neuroprotection for traumatic brain injury: translational challenges and emerging therapeutic strategies. Trends Pharmacol Sci 2010; 31(12):596-604.

35. Pan Z, Zhao Y, Huang W, Xiao Z, Li Z. Effect of progesterone administration on the prognosis of patients with severe traumatic brain injury: a meta-analysis of randomized clinical trials. Drug Des Devel Ther 2019; 13:265-273.

\section{Contribución de autores}

William Flórez Perdomo, https://orcid.org/0000-0002-6951-1277. Concepción, planteamiento pregunta PICO, estrategia de búsqueda, búsqueda sistemática de la información, recolección de datos y análisis estadístico, redacción y revisión del documento.

Miguel Durán, https://orcid.org/0000-0002-6043-9272. Concepción, PICO, estrategia de búsqueda, búsqueda sistemática de la información, recolección de datos y análisis estadístico, redacción y revisión del documento.

Edgar F Laiseca, https://orcid.org/0000-0002-5357-8274. Concepción, planteamiento pregunta PICO, estrategia de búsqueda, búsqueda sistemática de la información, recolección de datos y análisis estadístico, redacción y revisión del documento

Sergio A Serrato, https://orcid.org/0000-0002-4399-4864. Concepción, planteamiento pregunta PICO, estrategia de búsqueda, búsqueda sistemática de la información, recolección de datos y análisis estadístico, redacción y revisión del documento Mario F Quesada, https://orcid.org/0000-0001-5399-4596. Concepción, estrategia de búsqueda, búsqueda sistemática de la información, recolección de datos y análisis estadístico, redacción 
Anexo A. Estrategia de búsqueda

1.exp Progesterone/

2.exp Progestins/

3.exp Receptors, Progesterone/

4. $\exp$ Gonadal Steroid Hormones/

5.exp Estrogens/

6.exp Receptors, Estrogen/

7.(Progestin* or gestagen* ${ }^{*}$ or progestagen* or progestogen* or progestation ${ }^{*}$ or estrogen $\left.{ }^{*}\right)$.ab,ti.

8.((gender* or gonad* or sex*) adj3 hormon*).ab,ti.

9.((gender or Sex ${ }^{*}$ or hormon ${ }^{*}$ ) adj3 (differ ${ }^{*}$ or effect ${ }^{*}$ or influence ${ }^{*}$ or function* ${ }^{*}$ or recover $\left.)\right) . t i, a b$.

10.or/1-9

11.exp Craniocerebral Trauma/

12.exp Brain Edemal

13.exp Glasgow Coma Scale/

14.exp Glasgow Outcome Scale/

15.exp Unconsciousness/

16.exp Cerebrovascular Trauma/

17.((head or crani* or cerebr* or capitis or brain* or forebrain* or skull ${ }^{*}$ or hemispher* or intra-cran* or inter-cran*) adj5 (injur ${ }^{*}$ or trauma* or damag* or wound* or fracture* or contusion*)).ab,ti.

18.((head or crani* or cerebr* or brain* or intra-cran* or inter-cran*) adj5 (haematoma* or hematoma* or haemorrhag* or hemorrhag* or bleed* or pressure)).ti,ab.

19.(Glasgow adj (coma or outcome) adj (scale* or score $\left.{ }^{*}\right)$ ).ab,ti.

20."Rancho Los Amigos Scale".ti,ab.

21.("diffuse axonal injury" or "diffuse axonal injuries").ti,ab.

22.((brain or cerebral or intracranial) adj3 (oedema or edema or swell $\left.{ }^{\star}\right)$ ).ab,ti.

23.((unconscious ${ }^{\star}$ or coma* or concuss ${ }^{\star}$ or 'persistent vegetative state') adj3 (injur ${ }^{\star}$ or trauma* or damag $^{*}$ or wound* or fractu$\left.\left.\mathrm{re}^{*}\right)\right) \cdot \mathrm{ti}, \mathrm{ab}$.

24.exp coma/

25.or/11-24

26.randomi?ed.ab,ti.

27.randomized controlled trial.pt.

28.controlled clinical trial.pt.

29. placebo.ab.

30.clinical trials as topic.sh.

31.randomly.ab.

32.trial.ti.

33.or/26-32

34.(animals not (humans and animals)).sh.

35.33 not 34

36.25 and 35

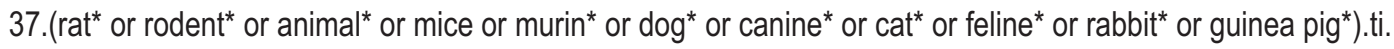

38.36 not 37

39.10 and 38 
Anexo B. Herramienta de la Colaboración Cochrane para evaluar el riego de sesgo. Tomado de Manual Cochrane 5.1.0. / Parte 2: Métodos generales para las revisiones. Cochrane capítulo 8: Evaluación del riesgo de sesgo en los estudios incluidos.

\begin{tabular}{|c|c|c|}
\hline Dominio & Descripción & Valoración de los revisores \\
\hline \multicolumn{3}{|l|}{ Sesgo de selección } \\
\hline Generación de la secuencia. & $\begin{array}{l}\text { Describir el método utilizado para generar la } \\
\text { secuencia de asignación con detalle suficiente } \\
\text { para permitir una evaluación de si la misma } \\
\text { produjo grupos comparables. }\end{array}$ & $\begin{array}{l}\text { Sesgo de selección (asignación sesgada a las } \\
\text { intervenciones) a causa de una generación } \\
\text { inadecuada de la secuencia de aleatorización. }\end{array}$ \\
\hline Ocultamiento de la asignación. & $\begin{array}{l}\text { Describir el método utilizado para ocultar la } \\
\text { secuencia de asignación con detalle suficiente } \\
\text { para determinar si las asignaciones a la } \\
\text { intervención se podían prever antes o durante el } \\
\text { reclutamiento. }\end{array}$ & $\begin{array}{l}\text { Sesgo de selección (asignación sesgada a las } \\
\text { intervenciones) a causa de una ocultación } \\
\text { inadecuada de las asignaciones antes de } \\
\text { asignarlas. }\end{array}$ \\
\hline \multicolumn{3}{|l|}{ Sesgo de realización } \\
\hline $\begin{array}{l}\text { Cegamiento de los participantes y del personal. } \\
\text { Se debería evaluar cada resultado principal (o } \\
\text { cada clase de resultado). }\end{array}$ & $\begin{array}{l}\text { Describir todas las medidas utilizadas, si se } \\
\text { utilizó alguna, para cegar a los participantes y al } \\
\text { personal del estudio al conocimiento de qué } \\
\text { intervención recibió un participante. Proporcionar } \\
\text { cualquier información con respecto a si el } \\
\text { cegamiento propuesto fue efectivo. }\end{array}$ & $\begin{array}{l}\text { Sesgo de realización a causa del conocimiento } \\
\text { por parte de los participantes y del personal } \\
\text { durante el estudio de las intervenciones } \\
\text { asignadas. }\end{array}$ \\
\hline \multicolumn{3}{|l|}{ Sesgo de detección } \\
\hline $\begin{array}{l}\text { Cegamiento de los evaluadores del resultado. } \\
\text { Se debería evaluar cada resultado principal (o } \\
\text { cada clase de resultado). }\end{array}$ & $\begin{array}{l}\text { Describir todas las medidas utilizadas, si se } \\
\text { utilizó alguna, para cegar a los evaluadores del } \\
\text { resultado del estudio al conocimiento de qué } \\
\text { intervención recibió un participante. }\end{array}$ & \\
\hline $\begin{array}{l}\text { Proporcionar cualquier información con respecto } \\
\text { a si el cegamiento propuesto fue efectivo. }\end{array}$ & $\begin{array}{l}\text { Sesgo de detección a causa del conocimiento } \\
\text { por parte de los evaluadores de los resultados } \\
\text { de las intervenciones asignadas. }\end{array}$ & \\
\hline \multicolumn{3}{|l|}{ Sesgo de desgaste } \\
\hline $\begin{array}{l}\text { Datos de resultado incompletos. Se debería } \\
\text { evaluar cada resultado principal (o cada clase de } \\
\text { resultado). }\end{array}$ & $\begin{array}{l}\text { Describir la compleción de los datos de } \\
\text { resultado para cada resultado principal, incluidos } \\
\text { los abandonos y las exclusiones del análisis. } \\
\text { Señalar si se describieron los abandonos y las } \\
\text { exclusiones, los números en cada grupo de } \\
\text { intervención (comparados con el total de } \\
\text { participantes asignados al azar), los motivos de } \\
\text { las deserciones/exclusiones cuando se } \\
\text { detallaron, y cualquier reinclusión en los análisis } \\
\text { realizada por los revisores. }\end{array}$ & $\begin{array}{l}\text { Sesgo de desgaste a causa de la cantidad, la } \\
\text { naturaleza o el manejo de los datos de resultado } \\
\text { incompletos. }\end{array}$ \\
\hline $\begin{array}{l}\text { Notificación selectiva de los resultados. Se } \\
\text { debería evaluar cada resultado principal (o cada } \\
\text { clase de resultado). }\end{array}$ & $\begin{array}{l}\text { Señalar cómo los revisores examinaron la } \\
\text { posibilidad de la notificación selectiva de los } \\
\text { resultados, y qué encontraron. }\end{array}$ & $\begin{array}{l}\text { Sesgo de notificación a causa de la notificación } \\
\text { selectiva de los resultados. }\end{array}$ \\
\hline \multicolumn{3}{|l|}{ Otros sesgos } \\
\hline Otras fuentes de sesgo. & $\begin{array}{l}\text { Señalar alguna inquietud importante acerca de } \\
\text { sesgo no abordada en los otros dominios del } \\
\text { instrumento. Si en el protocolo de la revisión se } \\
\text { preespecificaron preguntas/ítems particulares, } \\
\text { se deberían proporcionar las respuestas para } \\
\text { cada pregunta/ítem. }\end{array}$ & $\begin{array}{l}\text { Sesgo debido a otros problemas no abordados } \\
\text { en los apartados anteriores. }\end{array}$ \\
\hline
\end{tabular}


Anexo C. Estudios incluidos y excluidos

\section{Estudios incluidos}

\begin{tabular}{|c|c|c|c|c|c|c|}
\hline Estudios & Año & Tipo & Progesterona & Control & Intervención & Resultados \\
\hline Aboukhabar & 2012 & $\mathrm{RCT}$ & 50 & 50 & $\begin{array}{l}\text { Progesterona (IM): } 1 \mathrm{mg} / \mathrm{kg} \text { cada } 12 \\
\text { horas por } \\
5 \text { días }\end{array}$ & $\begin{array}{l}\text { Mortalidad a } 6 \text { meses, GOS, días de } \\
\text { estancia en UCl }\end{array}$ \\
\hline NETT GROUP & 2014 & $\mathrm{RCT}$ & 74 & 74 & $\begin{array}{l}\text { Progesterona (IV): } 0,71 \mathrm{mg} / \mathrm{kg} \text { en la } \\
\text { primera hora, luego } 0,5 \mathrm{mg} / \mathrm{kg} / \mathrm{h} \text { por } 3 \\
\text { días y } 0,125 \mathrm{mg} / \mathrm{kg} / \mathrm{h} \text { por } 24 \text { horas } \\
\text { adicionales }\end{array}$ & Mortalidad a 6 meses; GOS \\
\hline ProTECT & 2007 & $\mathrm{RCT}$ & 77 & 23 & $\begin{array}{l}\text { Progesterona (IV): } 0,71 \mathrm{mg} / \mathrm{kg} \text { en la } \\
\text { primera hora, luego a } 0,5 \mathrm{mg} / \mathrm{kg} \text { cada } \\
12 \text { horas por } 3 \text { días }\end{array}$ & $\begin{array}{l}\text { Mortalidad a 3- } 6 \text { meses, GOS, } \\
\text { Presión intracraneana }\end{array}$ \\
\hline Shakeri & 2013 & RCT & 38 & 38 & $\begin{array}{l}\text { Medroxiprogesterone tabletas : } 1 \\
\mathrm{mg} / \mathrm{kg} \text { cada } 12 \text { horas por } 5 \text { días }\end{array}$ & Mortalidad a 3 meses, GOS \\
\hline Shina & 2017 & $\mathrm{RCT}$ & 26 & 27 & $\begin{array}{l}\text { Progesterona (IM): } 1 \mathrm{mg} / \mathrm{kg} \text { cada } 12 \\
\text { horas por } 5 \text { días }\end{array}$ & $\begin{array}{l}\text { Mortalidad a } 6 \text { meses, GOS, } \\
\text { Independencia funcional }\end{array}$ \\
\hline SYNAPSE Trial & 2014 & $\mathrm{RCT}$ & 591 & 588 & $\begin{array}{l}\text { Progesterona (IV): } 0,71 \mathrm{mg} / \mathrm{kg} / \mathrm{h} \text { en la } \\
\text { primera hora, luego } 0,5 \mathrm{mg} / \mathrm{kg} / \mathrm{h} \text { por } 5 \\
\text { días }\end{array}$ & Mortalidad a 6 meses; GOS \\
\hline Xiao & 2008 & $\mathrm{RCT}$ & 82 & 77 & $\begin{array}{l}\text { Progesterona (IM): } 1 \mathrm{mg} / \mathrm{kg} \text { cada } 12 \\
\text { horas por } 5 \text { días }\end{array}$ & $\begin{array}{l}\text { Mortalidad a } 6 \text { meses, GOS, } \\
\text { Independencia funcional }\end{array}$ \\
\hline
\end{tabular}

RCT: ensayo clínico controlado aleatorizado (Randomized Clinical Trial) GOS: Glasgow Outcome Scale IM: intramuscular IV: intravenoso UCI: unidad de cuidados intensivos.

\section{Estudios excluidos}

\begin{tabular}{ll}
\hline Estudio & \multicolumn{1}{c}{ Razón de exclusión } \\
\hline Mofid, 2016 & No responde la pregunta fijada, evalúa su relación con los niveles de interleucinas \\
Xiao, 2007 & No evalúa discapacidad ni pronóstico neurológico \\
Aminmansour, 2012 & Evalúa progesterona + vitamina D \\
Raheja, 2016 & Evalúa niveles de marcadores séricos, no evalúa pronóstico ni mortalidad como desenlace \\
Wrigth, 2005 & $\begin{array}{l}\text { Este estudio evaluó la farmacocinética de la progesterona administrada por infusión intravenosa en pacientes } \\
\text { con TBI. No se presentaron datos sobre efectos terapéuticos. }\end{array}$
\end{tabular}

